This is the author's final, peer-reviewed manuscript as accepted for publication. The publisher-formatted version may be available through the publisher's web site or your institution's library.

\title{
Entanglement model of antibody viscosity
}

Jeremy D. Schmit, Feng He, Shradha Mishra, Randal R. Ketchem, Christopher E. Woods, and Bruce A. Kerwin

\section{How to cite this manuscript}

If you make reference to this version of the manuscript, use the following information:

Schmit, J. D., He, F., Mishra, S., Ketchem, R. R., Woods, C., E., \& Kerwin, B. A. (2014). Entanglement model of antibody viscosity. Retrieved from http://krex.ksu.edu

\section{Published Version Information}

Citation: Schmit, J. D., He, F., Mishra, S., Ketchem, R. R., Woods, C., E., \& Kerwin, B. A. (2014). Entanglement model of antibody viscosity. Journal of Physical Chemistry B, 118(19), 5044-5049.

Copyright: @ 2014 American Chemical Society

Digital Object Identifier (DOI): doi:10.1021/jp500434b

Publisher's Link: http://pubs.acs.org/doi/abs/10.1021/jp500434b

This item was retrieved from the K-State Research Exchange (K-REx), the institutional repository of Kansas State University. K-REx is available at http://krex.ksu.edu 


\title{
Entanglement Model of Antibody Viscosity
}

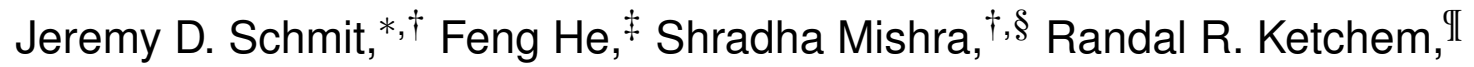 \\ Christopher E. Woods, ${ }^{\ddagger}$ and Bruce A. Kerwin*,\$
}

Department of Physics, Kansas State University, Manhattan, KS 66506, USA, Amgen, Inc., Department of Drug Product Development, Seattle, Washington, 98101, USA, and Amgen, Inc., Department of Biological Optimization, Seattle, Washington, 98101, USA

E-mail: schmit@phys.ksu.edu; bkerwin@amgen.com

*To whom correspondence should be addressed

$\dagger$ Kansas State University

†Dept. of Drug Product Development

IDept. of Biological Optimization

${ }^{\S}$ Current address: Dept. of Theoretical Studies, S N Bose National Centre for Basic Sciences, Kolkata, India 700098 


\begin{abstract}
Antibody solutions are typically much more viscous than solutions of globular proteins at equivalent volume fraction. Here we propose that this is due to molecular entanglements that are caused by the elongated shape and intrinsic flexibility of antibody molecules. We present a simple theory in which the antibodies are modeled as linear polymers that can grow via reversible bonds between the antigen binding domains. This mechanism explains the observation that relatively subtle changes to the interparticle interaction can lead to large changes in the viscosity. The theory explains the presence of distinct power law regimes in the concentration dependence of the viscosity as well as the correlation between the viscosity and the charge on the variable domain in our anti-streptavidin $\mathrm{IgG}_{1}$ model system.
\end{abstract}

keywords: formulation, theory, polymer, reptation, biotechnology

\title{
Introduction
}

Patient convenience is becoming an increasingly important aspect for formulation and delivery of biotherapeutics. While earlier biotherapeutics were dosed at microgram to milligram levels with frequent dosing schedules, antibody based drugs are generally dosed in the tens to hundreds of milligrams range. This is especially relevant as the frequency of dosing decreases, leading to the need for protein concentrations $>100 \mathrm{mg} / \mathrm{mL}$ that are compatible with autodelivery devices for patient self-injection. As antibody solutions become more concentrated, the viscosity of the solutions can increase dramatically leading to difficulty with manufacturing and delivery. This problem is especially true for monoclonal antibodies (mAbs) used for treating a wide array of diseases related to inflammation. ${ }^{1,2}$

Initially, therapeutic mAbs were developed with the binding affinities to targets as the exclusive consideration and little thought of formulation and syringeability with the idea that viscosity at high concentration could be reduced using $\mathrm{pH}$ and various excipients. ${ }^{2-13}$ More recently, the viscosity problem is addressed using high-throughput screening of formulation conditions. ${ }^{6,14} \mathrm{~A}$ 
better approach would be to tune the viscosity early in the development pipeline when the sequence can be used as a variable. Recent attempts at identifying sequence and specific residues responsible for inducing the viscosity at high protein concentrations have had limited success. ${ }^{15}$ Yadav et al. swapped sequences from the complementarity determining regions (CDR) in two otherwise identical antibodies, one with high and the other low viscosity, successfully lowering the viscosity of one $\mathrm{mAb}$ but not increasing the viscosity of the other. This suggests that bioinformatic approaches would have limited predictive power in identifying high-viscosity CDR loops and motivates the development of physics-based models.

In order to utilize the sequence as a variable to tune the viscosity, it will be necessary to understand precisely how mAb properties give rise to elevated viscosity. This understanding is a prerequisite for the detailed in silico models needed to predict the viscosity based on sequence and solvent conditions. Previous attempts to model antibody viscosity have employed empirical quasi-spherical representations. ${ }^{16-18}$ A common feature of these models is the presence of fitting parameters that greatly enhance the viscosity beyond that expected for a comparable solution of ideal spheres or ellipsoids. ${ }^{19}$ Due to the empirical nature of these formulas, they provide little insight into the microscopic events that give rise to the viscosity and are not suitable for predicting the effects of sequence or solvent changes. Several studies have implicated reversible proteinprotein interactions as the cause of elevated viscosity. ${ }^{9,11,12,20}$ Yet, it is unclear how these protein assemblies might affect the viscosity if the protein volume fraction remains unchanged. ${ }^{18}$

In this paper we make the opposite approximation of the rigid particle models and treat the antibodies as highly flexible molecules. The goal of this model is to determine if the elongated shape and intrinsic flexibility of antibodies are playing an important role in the viscosity. This approach is motivated by the observation that globular proteins lacking the large-scale flexibility of antibodies have much lower viscosities than antibodies at comparable volume fractions. ${ }^{21-24}$ Somewhat surprisingly, we find that antibody viscosities are consistent with reptation dynamics as would be expected in a semi-dilute solution of polymers. In such systems, topological constraints imposed by neighboring molecules restrict the lateral motion of molecules forcing them to relax 
via diffusion parallel to the polymer backbone. ${ }^{25}$ We expect that in concentrated antibody solutions the diffusion occurs primarily along the most extended molecular dimension, which would be along a contour connecting the two variable regions. This restricted mobility is exacerbated by protein-protein binding events which cause the antibodies to form more extended structures, thereby enhancing the potential for entanglements.

\section{Model}

Scaling theory gives the concentration dependence of viscosity. In order to model the effect of molecular flexibility on viscosity, we approximate each antibody as a featureless polymer with the polymer ends located at the antigen binding sites. At the concentration range of interest, above $100 \mathrm{mg} / \mathrm{ml}$, the center-to-center particle separation is comparable to the molecular size and the antibodies occupy a solution volume on the order of $10 \%$. Therefore, we adopt a viscosity model for a semi-dilute polymer solution. ${ }^{26}$ The viscosity is proportional to the product of the elastic modulus $G$ and the longest relaxation time $\tau,{ }^{27}$ which takes the following form in the semi-dilute regime $^{28-30}$

$$
\begin{aligned}
\eta & \propto G \tau \\
& \sim \eta_{s}\left(\frac{c}{c^{*}}\right)^{3 /(3 v-1)}
\end{aligned}
$$

where $\eta_{s}$ is the viscosity of the solvent, $v \simeq 3 / 5$ is the Flory exponent, $c$ is the segment concentration, and $c^{*}$ is the overlap concentration. Each antibody can be interpreted as a "polymer" of length $L=3$ segments corresponding to the three domains of the antibody molecule. Eq. (2) is expected to be valid when the length of the polymer greatly exceeds the monomer dimensions. After accounting for the self-association of the antibodies, our solution amount to a mixture of polymers of length $3 \leq L \lesssim 10$, which is still too short to satisfy the separation of length scales required for scaling theories like Eq. (2). Nevertheless, in the spirit of the "spherical cow" approximation 
we adopt this over-simplified model in the hope of gaining insights from an analytically tractable theory.

The overlap concentration for a solution of polymers is the point at which the random coil volumes, $\sim L^{3 v}$, occupy the full solution volume. A rough estimate of the antibody overlap concentration can be obtained from the monomer dimension, $\sim 15 \mathrm{~nm}$, yielding $c^{*} \sim 70 \mathrm{mg} / \mathrm{ml}$. The apparent coincidence of this value with the nonlinear increase in viscosity does not provide evidence for the polymer model as this is simply the concentration at which many-body interactions would be expected to dominate the viscosity, regardless of the mechanism. The overlap concentration declines with polymer length as the coil volumes expand to include more solvent. For polymers consisting of $L$ segments of size $b$, the overlap concentration is $c^{*} \sim L^{1-3 v} b^{-3} \cdot{ }^{30}$ Inserting this into Eq. (2) we have

$$
\eta \sim c^{3 /(3 v-1)} L^{3}
$$

From Eq. (3) we see that this viscosity should increase with concentration according to $c^{3.75}$ for $v \simeq 0.6$. Also from Eq. (3) it is apparent that the viscosity depends strongly on the polymer length $L$. The formation of reversible antibody-antibody complexes will lead to longer effective polymer lengths, thereby increasing the viscosity. This is an important feature of our model and contrasts strongly with quasi-spherical models. In quasi-spherical models the viscosity is a function of the solute volume fraction which changes minimally upon protein-protein association. To compute $L$ we adopt a 1D equilibrium polymerization model (see Figure 1). This calculation amounts to a discretized version of Cates' theory for the viscosity of 'living polymers'. ${ }^{31,32}$

Intermolecular binding leads to longer effective lengths and more entanglements. We assume that antibody-antibody binding events occur primarily between antigen binding domains resulting in the linear aggregates shown in Figure 1. This 'head-to-head' model is not an essential feature of our theory. In fact, for the small complex sizes we find after fitting to experimental data, a model describing head-to-tail binding would yield similar results. We expect that the binding sites, and hence the aggregate topology, will depend on the specific antibody under consideration. This will be discussed in more detail below. 


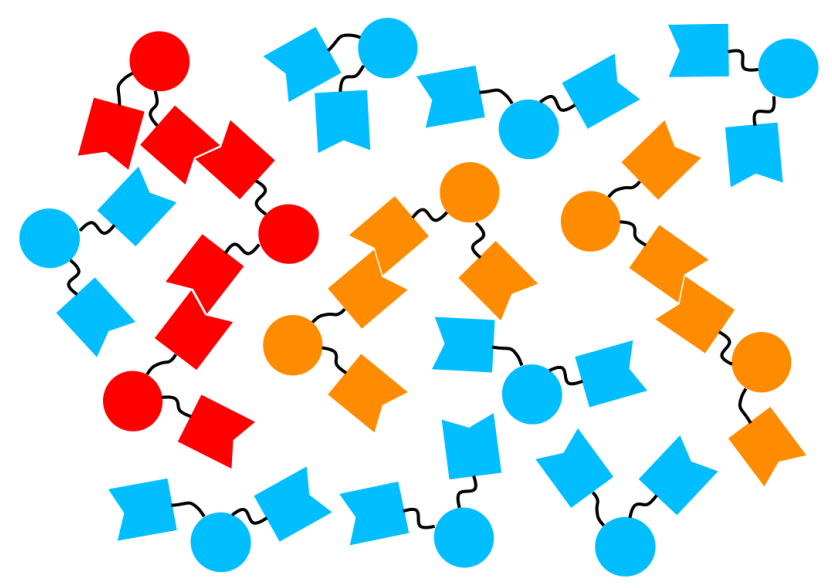

Figure 1: Cartoon of the linear aggregation model. Transient binding interactions between the variable domains causes the antibodies to link together into flexible chains. The entanglement of these chains results in a large viscosity. The figure shows monomeric proteins (blue), dimers (orange), and a trimer (red).

To calculate aggregate sizes in the antibody solution we construct the grand canonical partition function

$$
Q=\frac{1}{k} \sum_{n=1}^{\infty}\left(c_{1} k\right)^{n}=\frac{c_{1}}{1-c_{1} k} .
$$

Here we have defined the monomer state to have zero free energy so that the chemical potential is given by $\mu=k_{B} T \ln c_{1}$, where $c_{1}$ is the monomer concentration. The equilibrium constant for the formation of a $n$-mer is therefore $k^{n-1}$ where $-k_{B} T \ln k$ is the free energy of an intermolecular bond (strictly speaking, $k$ is the partition function over all potential intermolecular binding states). Eq. (4) is valid when the system is near equilibrium. This is exact for the dynamic light scattering (DLS) measurements of the viscosity that we employ here, however, methods that apply nonzero shear will drive the system out of equilibrium and it may be necessary to utilize a kinetic model for protein association.

The total protein concentration is given by

$$
c=\frac{1}{k} \sum_{n=1}^{\infty} n\left(c_{1} k\right)^{n}=c_{1} \frac{\partial Q}{\partial c_{1}}=\frac{c_{1}}{\left(1-c_{1} k\right)^{2}} .
$$

This expression can be inverted to find the monomer concentration as a function of the total protein 
concentration

$$
\begin{aligned}
c_{1} & =\frac{(2 k+1 / c) \pm \sqrt{(2 k+1 / c)^{2}-4 k^{2}}}{2 k^{2}} \\
& =\frac{1}{2 k^{2} c}(1+2 k c-\sqrt{4 k c+1}),
\end{aligned}
$$

where the lower sign has been selected to give the limit $c_{1} \rightarrow c$ at low concentrations.

To solve for the effective molecular size appearing in the expression for the viscosity, we find the average assembly size from Eq. (4), Eq. (5), and Eq. (7),

$$
\begin{aligned}
\langle N\rangle & =\frac{\frac{1}{k} \sum_{n=1}^{\infty} n\left(c_{1} k\right)^{n}}{Q}=\frac{c}{Q} \\
& =\frac{2 k c}{\sqrt{1+4 k c}-1} .
\end{aligned}
$$

To obtain an expression for the viscosity we insert Eq. (9) into Eq. (3). The result is

$$
\eta=A c^{3 /(3 v-1)}\left(\frac{2 k c}{\sqrt{1+4 k c}-1}\right)^{3}
$$

where $A$ is a constant of proportionality that will be obtained by fitting. Note that we have absorbed into $A$ a constant factor arising from the conversion from aggregation number to polymer length $3\langle N\rangle=L$

\section{Methods}

Preparation of $\mathbf{m A b}$ solutions Purified anti-streptavidin $\mathrm{IgG}_{1}$ monoclonal antibody solutions were dialyzed (1:1,000,000 volume ratio) against buffer solutions containing $20 \mathrm{mM}$ Sodium Acetate (pH 5.0), 25 mM Sodium Acetate ( $\mathrm{pH} 5.5), 10 \mathrm{mM}$ Histidine ( $\mathrm{pH} 6.0$ and $\mathrm{pH}$ 6.5) and 20 $\mathrm{mM}$ Histidine $(\mathrm{pH} 6.0)$ at $25^{\circ} \mathrm{C}$ using dialysis cassettes with a 10,000 MWCO (Thermo Scientific,

Rockford, IL). Each sample was concentrated by centrifugation using 10,000 MWCO Amicon Ultra-15 centrifugal concentration devices (Millipore Corporation, Billerica, MA) until a target 
protein concentration of $240 \mathrm{mg} / \mathrm{mL}$ was achieved. Concentrated mAb solutions were diluted by direct mixing with corresponding buffer (20 mM Sodium Acetate (pH 5.0), 25 mM Sodium Acetate ( $\mathrm{pH}$ 5.5), $10 \mathrm{mM}$ Histidine ( $\mathrm{pH} 6.0$ or 6.5) or $20 \mathrm{mM}$ Histidine ( $\mathrm{pH} \mathrm{6.0))} \mathrm{to} \mathrm{achieve} \mathrm{desired}$ protein concentrations with $\mathrm{NaOH}$ added when necessary to achieve the desired $\mathrm{pH}$.

Viscosity Measurement The viscosity of sample solutions was measured using DLS according to a previously developed method. ${ }^{8,33,34}$ Briefly, $59.4 \mu \mathrm{L}$ of each sample was spiked with 0.6 $\mu \mathrm{L}$ of $200 \mathrm{~nm}$ (nominal diameter) polystyrene beads (Thermo Fisher Scientific Inc., Waltham, MA). The diffusion of the beads was then measured by DynaPro ${ }^{\mathrm{TM}}$ Platereader $^{\mathrm{TM}}$ DLS system (Wyatt Technology, Santa Barbara, CA) using a 384-well microplate with all samples run in duplicate. The scattering signal collection was done through 10 consecutive acquisitions with each acquisition lasting 30 seconds. Prior to the measurements, samples were incubated at the specified temperatures for at least 30 minutes. In order to obtain the apparent size of the polystyrene beads, water viscosity was assumed to allow diffusion coefficient calculation based on DLS data. The solution microviscosity was then derived using the apparent radius of the beads, the theoretical nominal radius of the beads and the theoretical water viscosity at the specified temperature (for example, $1.0 \mathrm{cP}$ at $\left.20^{\circ} \mathrm{C}\right)$.

Fitting procedure To compare Eq. (10) to experimental data, we require two missing constants. The first is an overall proportionality factor $A$ that is not expected to vary with solution conditions or antibody sequence. The second parameter, $k$, captures the binding affinity and is expected to vary strongly between molecules and with solvent conditions. To account for the temperature dependence of the binding affinity we adopt the following form for $k(T)$

$$
k=M_{w}^{-1} e^{-(h-T s) / k_{B} T}
$$

where the units are $(\mathrm{ml} / \mathrm{mg})$ and $h$ and $s$ are fitting parameters that can be interpreted as the enthalpy and entropy for antibody-antibody association. The molecular weight factor $M_{w} \simeq 142 \mathrm{kDa}$ facilitates this interpretation by setting the reference concentration in $s$ to the standard value of $1 \mathrm{M}$. 
The parameters $A, h$, and $s$ were obtained using a two step process. We first obtained $A$ as follows. For a fixed value of $A$ we fit $k$ to each of the 96 viscosity vs. concentration curves in Figure 2 and computed the sum of square errors. This process was repeated varying $A$ to obtain the optimal value $A=5.4 \times 10^{-8} \mathrm{cP}(\mathrm{mg} / \mathrm{ml})^{-3.75}$. Next, for each set of $\mathrm{pH}$ and salt conditions (each panel in Figure 2), Eq. (11) was fitted to the optimal $k$ values for each of the six temperatures. This reduced the six unique $k$ values to two parameters $(h$ and $s$ ). In total, there are $16 \times 2+1=33$ free parameters that are used to fit the 96 curves in Figure 2.

\section{Results and Discussion}
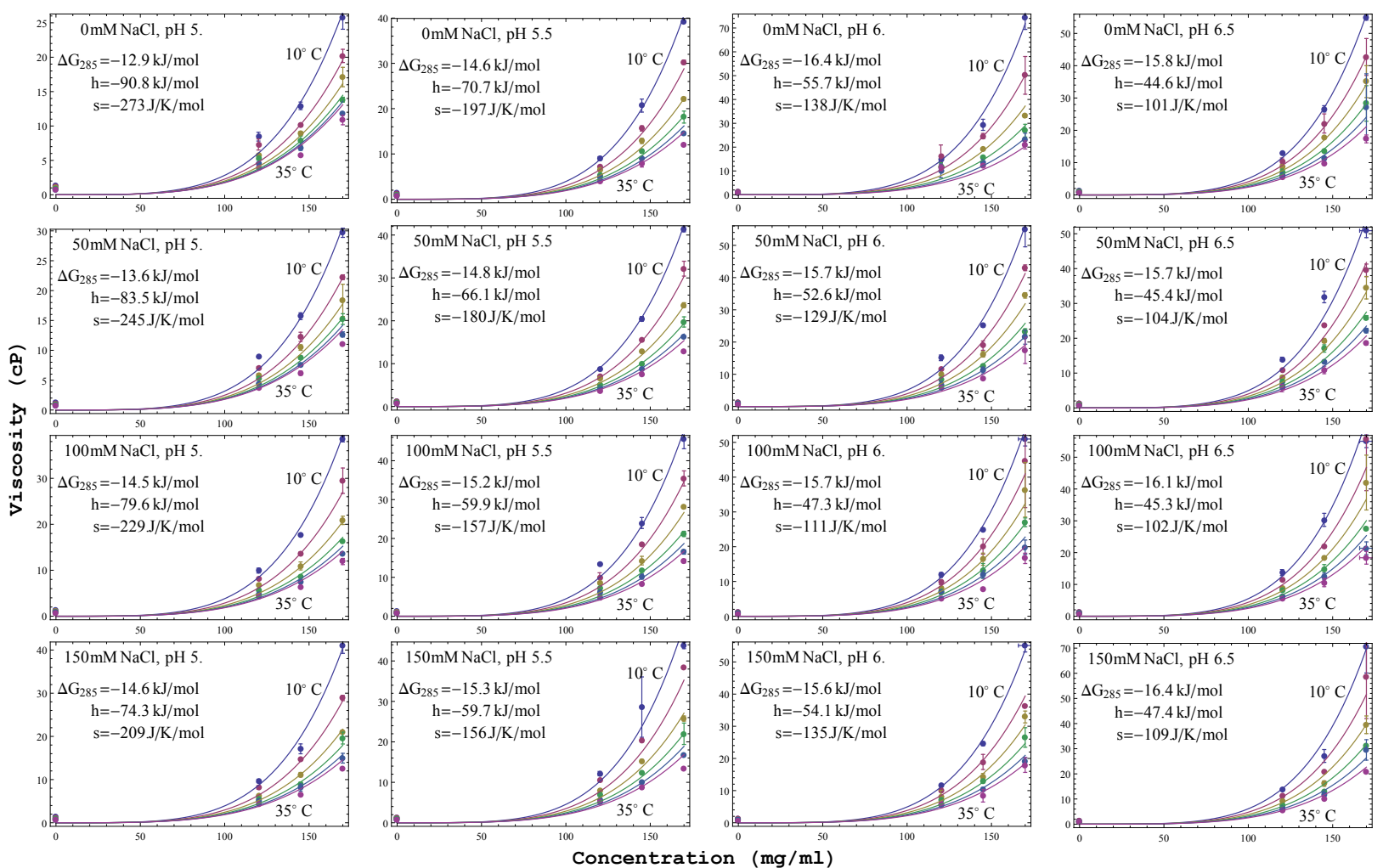

Figure 2: Fits of Eq. (10) to DLS measurements of mAb viscosity. Free energy parameters Eq. (11) are shown for each set of $\mathrm{pH}$ and salt conditions along with the binding free energy at $285 \mathrm{~K}$. Colors indicate temperature 10C (dark blue), 15C (maroon), 20C (yellow), 25C (green), 30C (light blue), $35 \mathrm{C}$ (violet). Horizontal error bars denote conditions for which there was only one usable replicate.

Small complex sizes are sufficient to explain the increase in viscosity. The fits to Eq. (10) 
are shown in Figure 2. The fitted binding energies are relatively modest, ranging from about $-2.5 k_{B} T$ to $-7 k_{B} T$ over the parameter space (Figure 3). At the highest concentration of 170 $\mathrm{mg} / \mathrm{ml}$, these binding energies correspond to average aggregate sizes of $\langle N\rangle \simeq 1$ under the least viscous conditions to $\langle N\rangle \simeq 1.8$ under the most viscous conditions ( $L=3-5.4$ domains). We note that the model's overestimation of the viscosity under the least viscous conditions (e.g. $\mathrm{pH} 5.0$ with $0 \mathrm{mM} \mathrm{NaCl}$ ) is suggestive that the obtained binding energies are insufficiently attractive and the overall prefactor $A$ should be correspondingly smaller. In fact, re-fitting the data using a value of $A$ that has been reduced by half fixes these errors in the low viscosity conditions and yields binding energies that are greater by 2 to $3 \mathrm{~kJ} / \mathrm{mol}$. While this fit has a visual quality similar to that in Figure 2, the sum of square errors is much higher due to the large weight given to the high viscosity data points in the numerical fitting procedure. This compensation between the fitted values of $A$ and the binding free energies means that there is considerable uncertainty in the free energies. Therefore, our primary message is that the viscosity is qualitatively consistent with molecular entanglements and that the antibody complexes that cause this are most likely small (2-4 molecules).

It is not known whether the fitted binding energies correspond to a single dominant bound state or a heterogeneous ensemble of states. Without this information it is difficult to interpret the effects of salt and $\mathrm{pH}$. However, the observed binding energies are consistent with the simple model shown in Figure 1 where the interactions occur primarily between the variable antigen binding domains $\left(\mathrm{F}_{\mathrm{v}}\right)$. The calculated charge on the $\mathrm{F}_{\mathrm{v}}$ is plotted in Figure $3 \mathrm{~b}$. The reduction in net charge from approximately +5 to +3 as the $\mathrm{pH}$ increases from 5.0 to 6.5 parallels the tendency for more favorable binding energies over the same range. This observation that the viscosity depends on the local charge density of the aggregation-prone regions explains the inconsistent correlation between electroviscous effects and measured antibody viscosity. ${ }^{17,35,36}$ In Figure 3 a there are several deviations from the expected monotonic relationship between the viscosity and the $\mathrm{pH}$ or salt concentration, most notably at $\mathrm{pH}$ 6.0. These are most likely due to small patches of charge complementarity that appear with the changing ionization states. Another possibility is that the non-monotonicity is an 
artifact of multiple competing bound states that have differing responses to the $\mathrm{pH}$. In any case, these effects are minor compared to the overall trend observed in Figure 3.
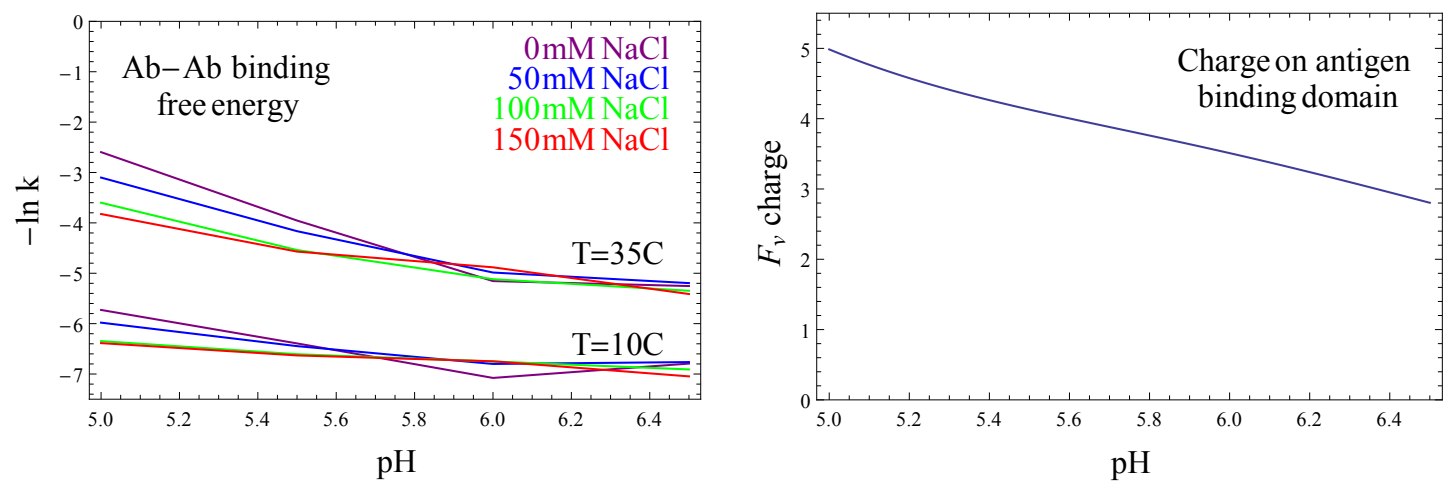

Figure 3: (left) Effect of $\mathrm{pH}$ and salt concentration on the free energy of antibody-antibody association. The free energy (plotted in units of $k_{B} T$ ) becomes more attractive as the $\mathrm{pH}$ is increased, which corresponds to a reduction in the net charge on the variable region. (right) Net charge on the variable region calculated using the pKa set of Grimsley et al. ${ }^{37}$

The viscosity shows three power law regimes. Eq. (10) predicts two different power laws for the effect of antibody concentration on viscosity. At low concentrations $c k \ll 1$ the factor in the parentheses is independent of concentration and the viscosity scales like $\eta \sim c^{3 /(3 v-1)} \sim c^{3.75}$. However, at higher concentrations the effective molecule size, Eq. (9), grows as $c^{1 / 2}$ suggesting that the viscosity should scale as $c^{5.25}$. Although it is not predicted by the model, a third concentration regime with a linear dependence will also be present in the dilute regime when the molecules are non-interacting.

The anti-streptavidin system does, in fact, show three power law regimes, although the exponents differ somewhat from the theoretical prediction (Figure 4). In the low concentration regime this deviation simply indicates that the experiments did not adequately sample concentrations within the linear regime. More interestingly, the exponents $\sim 2.7$ and $\sim 4.7$ observed at intermediate and high concentrations, respectively, are lower than the predicted values. This is not surprising since even ideal polymer systems show deviations from the predicted exponent of 3.75, although in these cases the exponent usually falls between 3 and $4 .{ }^{38}$ Another explanation for this discrepancy is that our antibody 'polymers' are too short to satisfy the scaling limit required in 
the derivation of Eq. (2). Our observed exponents are consistent with a slightly larger value of the Flory exponent $v \simeq 0.7$, which is reasonable since antibodies are too flexible to have a radius of gyration that scales like a rigid rod, $R_{g} \propto L^{1}$, yet not flexible enough to exhibit true Flory scaling, $R_{g} \propto L^{0.6}$. While this is suggestive that the fits in Figure 2 could be improved with a larger value of $v$, this is complicated by the fact that the optimal value of $v$ will vary with conditions. This is because the limit $v \rightarrow 0.6$ must be obtained when the antibody complexes grow very large.

Anti-streptavidin viscosity is not sensitive to the model binding site location. A potential pitfall for our model is the assumption of linear aggregation via head-to-head binding. In practice, antibodies vary widely and it is expected that different molecules will differ in their preferred intermolecular binding sites. This can have profound effects on the viscosity of the solution since binding interactions between the constant and antigen binding domains will lead to the formation of branched assemblies. In our anti-streptavidin model system branching is unlikely to play a role because the complex sizes are sufficiently small that the assumption of a linear assembly is always reasonable. In polymer systems branches exponentially increase the relaxation time due to the low probability of retracting the arms. ${ }^{27}$ In the case of a branched antibody assembly, which would be expected in systems where interactions between the constant and variable domains are the dominant mode of association, the arm retraction mechanism is unlikely to occur due to the limited flexibility of the antibodies. Therefore, the relaxation time would be determined by the timescale for the formation and breakage of the antibody-antibody contacts. We have neglected this timescale in the present treatment, but it could provide a significant reduction to the relaxation time, particularly in sheared systems. This effect will be explored in future work.

\section{Summary}

We have presented a simple theory for the viscosity of antibody solutions in which the antibodies are modeled as linear polymers. This model shows that the solution viscosity is consistent with molecular entanglements that are exacerbated when the molecules bind together to form larger 


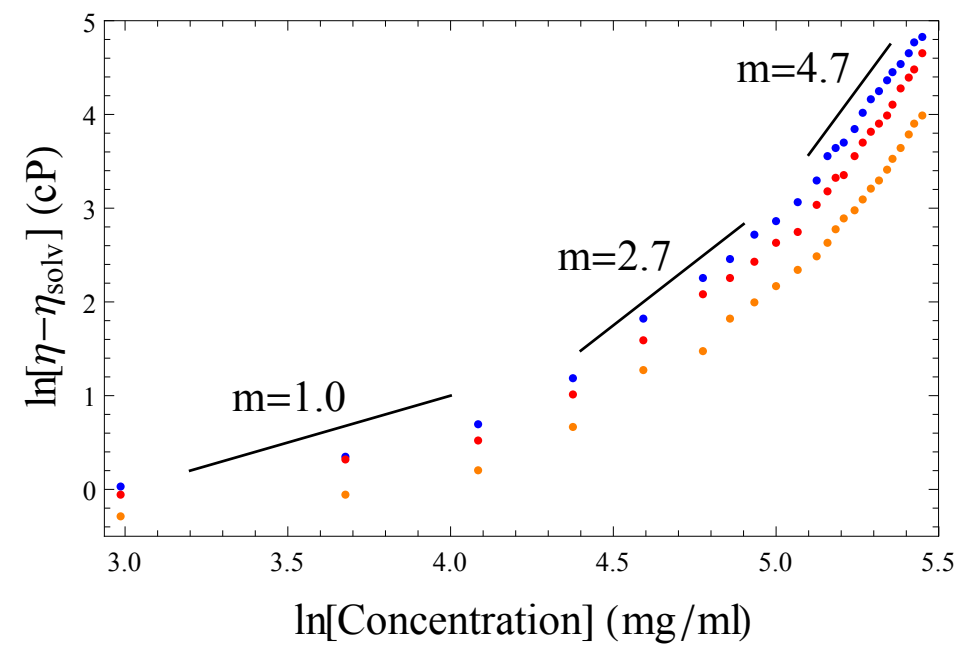

Figure 4: Log-Log plot of $\mathrm{mAb}$ viscosity showing three power law regimes as a function of protein concentration at $\mathrm{pH} 6.4$ and $0 \mathrm{mM} \mathrm{NaCl}$. Colors denote temperature as follows: $10^{\circ} \mathrm{C}$ (blue), $15^{\circ} \mathrm{C}$ (red), $25^{\circ} \mathrm{C}$ (yellow).

complexes. We hope that simple models like this one will motivate more detailed atomistic models for predicting antibody viscosity.

Acknowledgements JDS would like to thank L. Weaver and F. Pincus for valuable discussions. This work was supported by KSU startup funds and Amgen, Inc.

\section{References}

(1) Shire, S. J. Formulation and Manufacturability of Biologics. Curr. Opin. Biotech. 2009, 20, 708-714.

(2) Jezek, J.; Rides, M.; Derham, B.; Moore, J.; Cerasoli, E.; Simler, R.; Perez-Ramirez, B. Viscosity of Concentrated Therapeutic Protein Compositions. Adv. Drug Delivery Rev. 2011, 63, 1107-1117.

(3) Saito, S.; Hasegawa, J.; Kobayashi, N.; Kishi, N.; Uchiyama, S.; Fukui, K. Behavior of Monoclonal Antibodies: Relation Between the Second Virial Coefficient (B (2)) at Low Con- 
centrations and Aggregation Propensity and Viscosity at High Concentrations. Pharm. Res. 2012, 29, 397-410.

(4) Guo, Z.; Chen, A.; Nassar, R. A.; Helk, B.; Mueller, C.; Tang, Y.; Gupta, K.; Klibanov, A. M. Structure-Activity Relationship for Hydrophobic Salts as Viscosity-Lowering Excipients for Concentrated Solutions of Monoclonal Antibodies. Pharm. Res. 2012, 29, 3102-3109.

(5) Yadav, S.; Shire, S. J.; Kalonia, D. S. Viscosity Behavior of High-Concentration Monoclonal Antibody Solutions: Correlation with Interaction Parameter and Electroviscous Effects. $J$. Pharm. Sci. 2012, 101, 998-1011.

(6) Connolly, B. D.; Petry, C.; Yadav, S.; Demeule, B.; Ciaccio, N.; Moore, J. M. R.; Shire, S. J.; Gokarn, Y. R. Weak Interactions Govern the Viscosity of Concentrated Antibody Solutions: High-Throughput Analysis Using the Diffusion Interaction Parameter. Biophys. J. 2012, 103, 69-78.

(7) Du, W.; Klibanov, A. M. Hydrophobic Salts Markedly Diminish Viscosity of Concentrated Protein Solutions. Biotechnol. Bioeng. 2011, 108, 632-636.

(8) He, F.; Woods, C. E.; Litowski, J. R.; Roschen, L. A.; Gadgil, H. S.; Razinkov, V. I.; Kerwin, B. A. Effect of Sugar Molecules on the Viscosity of High Concentration Monoclonal Antibody Solutions. Pharm. Res. 2011, 28, 1552-1560.

(9) Yadav, S.; Liu, J.; Shire, S. J.; Kalonia, D. S. Specific Interactions in High Concentration Antibody Solutions Resulting in High Viscosity. J. Pharm. Sci. 2010, 99, 1152-1168.

(10) Chari, R.; Jerath, K.; Badkar, A. V.; Kalonia, D. S. Long- and Short-range Electrostatic Interactions Affect the Rheology of Highly Concentrated Antibody Solutions. Pharm. Res. 2009, 26, 2607-2618.

(11) Kanai, S.; Liu, J.; Patapoff, T. W.; Shire, S. J. Reversible Self-Association of a Concentrated 
Monoclonal Antibody Solution Mediated by Fab-Fab Interaction that Impacts Solution Viscosity. J. Pharm. Sci. 2008, 97, 4219-4227.

(12) Liu, J.; Nguyen, M. D. H.; Andya, J. D.; Shire, S. J. Reversible Self-Association Increases the Viscosity of a Concentrated Monoclonal Antibody in Aqueous Solution. J. Pharm. Sci. 2005, 94, 1928-1940.

(13) Patel, A. R.; Kerwin, B. A.; Kanapuram, S. R. Viscoelastic Characterization of High Concentration Antibody Formulations Using Quartz Crystal Microbalance with Dissipation Monitoring. J. Pharm. Sci. 2009, 98, 3108-3116.

(14) He, F.; Woods, C. E.; Trilisky, E.; Bower, K. M.; Litowski, J. R.; Kerwin, B. A.; Becker, G. W.; Narhi, L. O.; Razinkov, V. I. Screening of Monoclonal Antibody Formulations Based on High-throughput Thermostability and Viscosity Measurements: Design of Experiment and Statistical Analysis. J. Pharm. Sci. 2011, 100, 1330-1340.

(15) Yadav, S.; Sreedhara, A.; Kanai, S.; Liu, J.; Lien, S.; Lowman, H.; Kalonia, D. S.; Shire, S. J. Establishing a Link Between Amino Acid Sequences and Self-associating and Viscoelastic Behavior of Two Closely Related Monoclonal Antibodies. Pharm. Res. 2011, 28, 1750-1764.

(16) Monkos, K.; Turczynski, B. A Comparative Study on Viscosity of Human, Bovine and Pig IgG Immunoglobulins in Aqueous Solutions. Int. J. Biol. Macromol. 1999, 26, 155-159.

(17) Salinas, B. A.; Sathish, H. A.; Bishop, S. M.; Harn, N.; Carpenter, J. F.; Randolph, T. W. Understanding and Modulating Opalescence and Viscosity in a Monoclonal Antibody Formulation. J. Pharm. Sci. 2010, 99, 82-93.

(18) Minton, A. P. Hard Quasispherical Particle Models for the Viscosity of Solutions of Protein Mixtures. J. Phys. Chem. B 2012, 116, 9310-9315.

(19) Santamaría-Holek, I.; Mendoza, C. I. The Rheology of Concentrated Suspensions of Arbitrarily-shaped Particles. J. Colloid Interface Sci. 2010, 346, 118-126. 
(20) Yadav, S.; Laue, T. M.; Kalonia, D. S.; Singh, S. N.; Shire, S. J. The Influence of Charge Distribution on Self-Association and Viscosity Behavior of Monoclonal Antibody Solutions. Mol. Pharm. 2012, 9, 791-802.

(21) Monkos, K. Viscometric Study of Human, Bovine, Equine and Ovine Haemoglobin in Aqueous Solution. Int. J. Biol. Macromol. 1994, 16, 31-35.

(22) Monkos, K. Viscosity of Bovine Serum Albumin Aqueous Solutions as a Function of Temperature and Concentration. Int. J. Biol. Macromol. 1996, 18, 61-68.

(23) Monkos, K. Concentration and Temperature Dependence of Viscosity in Lysozyme Aqueous Solutions. Biochim. Biophys. Acta, Protein Struct. 1997, 1339, 304-310.

(24) Monkos, K. Viscosity Analysis of the Temperature Dependence of the Solution Conformation of Ovalbumin. Biophys. Chem. 2000, 85, 7-16.

(25) de Gennes, P.-G. Scaling Concepts in Polymer Physics; Cornell University Press: Ithaca, 1979.

(26) Zarraga, I. E.; Taing, R.; Zarzar, J.; Luoma, J.; Hsiung, J.; Patel, A.; Lim, F. J. High Shear Rheology and Anisotropy in Concentrated Solutions of Monoclonal Antibodies. J. Pharm. Sci. 2013, 102, 2538-2549.

(27) Rubinstein, M.; Colby, R. H. Polymer Physics (Chemistry); Oxford University Press: Oxford, 2003.

(28) De Gennes, P. G. Dynamics of Entangled Polymer Solutions. I. The Rouse Model. Macromolecules 1976, 9, 587-593.

(29) De Gennes, P. G. Dynamics of Entangled Polymer Solutions. II. Inclusion of Hydrodynamic Interactions. Macromolecules 1976, 9, 594-598.

(30) Doi, M.; Edwards, S. F. The Theory of Polymer Dynamics (International Series of Monographs on Physics); Oxford University Press: Oxford, 1988. 
(31) Cates, M. E. Reptation of Living Polymers: Dynamics of Entangled Polymers in the Presence of Reversible Chain-Scission Reactions. Macromolecules 1987, 20, 2289-2296.

(32) Cates, M. Dynamics of Living Polymers and Flexible Surfactant Micelles : Scaling Laws for Dilution. J. Phys. 1988, 49, 1593-1600.

(33) He, F.; Becker, G. W.; Litowski, J. R.; Narhi, L. O.; Brems, D. N.; Razinkov, V. I. Highthroughput Dynamic Light Scattering Method for Measuring Viscosity of Concentrated Protein Solutions. Anal. Biochem. 2010, 399, 141-143.

(34) Samra, H. S.; He, F. Advancements in High Throughput Biophysical Technologies: Applications for Characterization and Screening During Early Formulation Development of Monoclonal Antibodies. Mol. Pharm. 2012, 9, 696-707.

(35) Saluja, A.; Badkar, A. V.; Zeng, D. L.; Nema, S.; Kalonia, D. S. Application of Highfrequency Rheology Measurements for Analyzing Protein-Protein Interactions in High Protein Concentration Solutions Using a Model Monoclonal Antibody (IgG2). J. Pharm. Sci. 2006, 95, 1967-1983.

(36) Yadav, S.; Shire, S. J.; Kalonia, D. S. Factors Affecting the Viscosity in High Concentration Solutions of Different Monoclonal Antibodies. J. Pharm. Sci. 2010, 99, 4812-4829.

(37) Grimsley, G. R.; Scholtz, J. M.; Pace, C. N. A Summary of the Measured pK Values of the Ionizable Groups in Folded Proteins. Protein Sci. 2009, 18, 247-251.

(38) Graessley, W. W. The Entanglement Concept in Polymer Rheology; Advances in Polymer Science; Springer-Verlag: Berlin/Heidelberg, 1974; Vol. 16. 


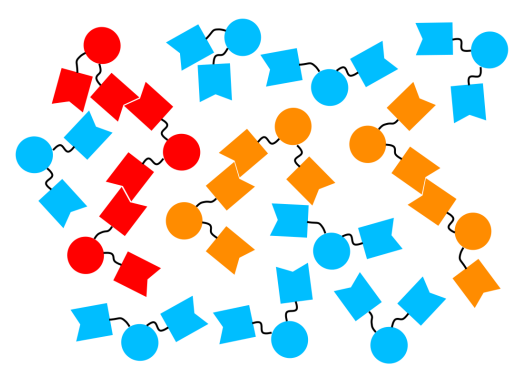

